\title{
Province-specific smoking-attributable cancer mortality in China 2013
}

\author{
Linjie Yu' ${ }^{1}$ Junxia Cheng ${ }^{1}$, Xiaoli Cui ${ }^{2}$, Jianbing Wang $^{3}$
}

\begin{abstract}
INTROduction Province-specific initiatives are at the forefront of tobacco control but limited studies have provided province-specific assessment of smoking-attributable cancer burden in China.

METHODS We estimated the fraction of total and site-specific cancer mortality attributable to tobacco smoking in 31 provinces in mainland China. The population attributable fractions (PAFs) for cancer deaths due to smoking were calculated by Levin's formula using province-specific smoking prevalence data around 1998 (assuming a 15-year latency time) and relative risks from cohort studies and meta-analyses. The $95 \%$ confidence intervals (CIs) of PAFs were calculated by a Delta method. Cancer deaths were abstracted from cancer registry data of the 31 provinces in mainland China in 2013.

RESULTS Overall, smoking contributed to a total of 421566 cancer deaths in mainland China in 2013 (19.46\% of all cancer deaths), with 400701 of these deaths occurring in men $(29.34 \%)$ and $20865(2.61 \%)$ in women. The population attributable fractions ranged from $15.56 \%$ (95\% CI: $9.12-$ $21.82 \%$ ) in Tibet to $35.09 \%$ (95\% CI: $25.68-45.83 \%$ ) in Guizhou among men, and from $0.28 \%$ (95\% CI: $0.00-0.64 \%$ ) in Hainan to $10.44 \%$ (95\% CI: $4.86-16.32 \%$ ) in Jilin among women. Cancers of lung and liver were the two main smoking-attributable cancers for both men and women.

CONCLUSIONS Tobacco smoking was responsible for nearly $20 \%$ of all cancer deaths in mainland China, but the proportion of cancer deaths attributable to smoking varied substantially across provinces. More effective programs and innovative new strategies for local tobacco control are warranted to reduce the future burden of smoking-related cancers in all provinces of mainland China.
\end{abstract}

\author{
AFFILIATION \\ 1 Department of Epidemiology and \\ Biostatistics, School of Public Health, \\ Zhejiang University, Hangzhou, People's \\ Republic of China \\ 2 Department of Gynecologic \\ Oncology, Cancer Hospital of China \\ Medical University, Liaoning Cancer \\ Hospital \& Institute, Shenyang, \\ People's Republic of China \\ 3 Department of Epidemiology and \\ Biostatistics, The Children's Hospital, \\ National Clinical Research Center \\ for Child Health, Zhejiang University \\ School of Medicine, Hangzhou, \\ People's Republic of China
}

\section{CORRESPONDENCE TO}

Xiaoli Cui. Department of Gynecologic Oncology, Cancer Hospital of China Medical University, Liaoning Cancer Hospital \& Institute, No. 44 Xiaoheyan Road, Dadong District, Shenyang, 110042, Liaoning Province, People's Republic of China. E-mail: cuixllxx@sina.com

KEYWORDS

smoking, cancer, population attributable fraction, China

Received: 22 November 2019

Revised: 30 April 2020

Accepted: 4 May 2020

\section{INTRODUCTION}

Cancer has become a leading cause of death in China in recent decades ${ }^{1}$. A number of epidemiology studies have demonstrated that most cancers are attributable to a modifiable lifestyle and environmental risk factors, among which smoking is the largest preventable one $^{2-5}$. Smoking, with a long-term adverse impact on health, has taken a great toll, of about 1 million deaths per year, on China and this is expected to exceed 3 million in $2050^{6}$. China is the world's largest producer and consumer of tobacco, with a population of 1.4 billion it has more than 301 million current smokers ${ }^{7}$. Due to substantial geographical variation in smoking prevalence, the burden of smoking related cancer across provinces remains unclear. In a previous study, we reported for the first time a systematic evaluation of the number of cancer cases and deaths attributable to carcinogens in 2005 in China ${ }^{8}$. In that study, we calculated the cancer cases and deaths attributable to smoking at the national level, but not at the provincial level. It is valuable to assess the local cancer burden caused by tobacco use as province-level initiatives 
are at the forefront of tobacco control, as indicated in the Lortet-Tieulent et al. ${ }^{9}$ study. However, most studies estimating smoking-related cancer mortality have been at the national level, and limited studies exist at the provincial level ${ }^{10-13}$.

Here, we provide an additional systematic assessment of cancer deaths attributable to smoking at the provincial level in China in 2013, aiming to provide scientific evidence for local policy makers to take effective action in tobacco control and cancer prevention.

\section{METHODS}

\section{Overview}

We estimate the province-specific proportion of cigarette smoking-related cancer mortality using similar methods in previous reports ${ }^{8,10}$. We selected 10 cancers caused by cigarette smoking that have been classified established targets of the carcinogenicity of tobacco by the International Agency for Research on Cancer (IARC) including: cancers of mouth/pharynx/ larynx, nasopharynx, lung, stomach, liver, esophagus, pancreas, colorectum, bladder, and kidney.

\section{Cancer deaths}

Data on cancer deaths in China in 2013 were based on the 255 qualified cancer registries distributed in the 31 provinces (autonomous regions and municipalities) of the annual cancer report of National Cancer Center, covering 226494490 of the population (including 114860339 males and 111634151 females) that accounted for $16.65 \%$ of the national population in 2013. In each province, cancer death rates were calculated by age group, sex, and cancer site. The death rates with sex-specific, age-specific and province-specific populations in 2013 were extrapolated to estimate the number of cancer deaths in each province. Finally, cancer death cases were calculated by summing the cases across all age groups and cancer sites in each province. This study is based on previously published data and does not include new human data that require again ethical approval and consent. The authors assume that the data source studies were conducted after ethical approval and consent, and in accordance with the Declaration of Helsinki 1975. The authors can confirm that all relevant data are included in the article and materials are available on request from the corresponding author.

\section{Latency time and smoking prevalence}

The occurrence of current cancers always reflects the past patterns of sustained smoking exposure, therefore there is a latency period between exposure and cancer. To date, no accurate studies are available to define the specific latency time between smoking and cancers. A report of World Health Organization (WHO) in 2000 suggested a latency time of 15 years ${ }^{14}$. Therefore, we used the smoking prevalence data of 1998.

Our study used a linear interpolation method to estimate provincial smoking prevalence in 1998 of data from two national surveys, in 1996 and $2002^{15,16}$, based on the previous study ${ }^{10}$. In our study, smoking was identified as 'continuous or cumulative smoking of at least one cigarette every day for 6 months or more during the lifetime ${ }^{\prime 17}$. Accordingly, we used the overall smoking status to represent smoking exposure, irrespective of current or former smoking status, type, amount, and duration of smoking.

\section{Relative risk}

Data on relative risks ( $R R s$ ) were obtained from different sources, including PubMed and China National Knowledge Infrastructure (CNKI), in Chinese or English. High-quality meta-analyses or large-scale pooled analyses from the Chinese population were given the highest priority for RRs between smoking and specific types of cancer mortality, followed by meta-analyses from Asian populations. For most cancers associated with smoking, the RRs used in our study were obtained from a large-scale pooled analysis of smoking and cancer in populations of China and South Korea ${ }^{18}$. However, RR for smoking and kidney cancer mortality in men was from a meta-analysis among the Asian population $^{19}$, and RR for smoking and esophageal cancer in women was abstracted from a prospective study in Linxian, China ${ }^{20}$ (Table 1 ).

\section{Statistical analysis}

Population attributable fraction (PAF) is defined as the proportional reduction of disease incidence or mortality in a population that would occur if exposure to a risk factor is reduced to an alternative ideal exposure scenario (e.g. no tobacco use) ${ }^{21}$. PAF was calculated according to Levin's formula, in which $\mathrm{p}$ represents the prevalence of exposure to the risk 
Table 1. Relative risks of site-specific smoking-related cancers in China 2013

\begin{tabular}{|c|c|c|c|c|c|c|}
\hline \multirow[t]{2}{*}{ Cancer sile } & \multirow{2}{*}{$\begin{array}{l}\text { ICD-10 } \\
\text { codle }\end{array}$} & \multirow[t]{2}{*}{ Study a } & \multirow[t]{2}{*}{ Design } & \multirow[t]{2}{*}{ Age (years) } & \multicolumn{2}{|c|}{$R R\left(95^{\circ} \circ \mathrm{CI}\right)$} \\
\hline & & & & & Ven & Women \\
\hline Mouth/larynx/ & $\mathrm{C} 00-\mathrm{C} 10$ & [18] & Pooled-analysis & $\geq 45$ & $1.95(1.51-2.5)$ & $1.99(1.11-3.59)$ \\
\hline Pharynx & C12-C14 & & & & & \\
\hline Stomach & C15 & [18] & Pooled-analysis & $\geq 45$ & $1.43(1.24-1.64)$ & $1.14(1.08-1.52)$ \\
\hline Colorectal & C18-C20 & [18] & Pooled-analysis & $\geq 45$ & $1.13(0.93-1.37)$ & $1.40(1.08-1.83)$ \\
\hline Liver & C22 & [18] & Pooled-analysis & $\geq 45$ & $1.35(1.19-1.53)$ & $1.75(1.05-2.84)$ \\
\hline Pancreas & $\mathrm{C} 25$ & [18] & Pooled-analysis & $\geq 45$ & $1.18(0.75-1.86)$ & $1.65(1.08-2.53)$ \\
\hline Lung & C33-C34 & [18] & Pooled-analysis & $\geq 45$ & $3.56(2.45-5.16)$ & $3.34(2.29-4.86)$ \\
\hline Bladder & C67 & [18] & Pooled-analysis & $\geq 45$ & $1.97(1.26-3.06)$ & $1.41(0.56-3.52)$ \\
\hline Kidney & C64 & [19] & Meta-analysis & - & $1.11(0.85-1.47)$ & $1.11(0.85-1.47)$ \\
\hline Nasopharynx & $\mathrm{C} 11$ & [18] & Pooled-analysis & $\geq 45$ & $2.22(1.42-3.49)$ & $2.22(1.42-3.49)$ \\
\hline \multirow[t]{2}{*}{ Esophagus } & $\mathrm{C} 15$ & [18] & Pooled-analysis & $\geq 45$ & $1.54(0.66-3.57)$ & - \\
\hline & & {$[20]$} & Prospective study & $40-69$ & - & $1.34(1.16-1.54)$ \\
\hline
\end{tabular}

a Zheng et al. ${ }^{18}$, Cumberbatch et al. ${ }^{19}$ and Tran et al. ${ }^{20}$. b RR was derived from the Asian population. c RR for female esophageal cancer associated with smoking was obtained from a prospective study in Linxian, China ${ }^{20}$.

factor in the total population, and RR represents the relative risk of a risk factor, where:

$$
\mathrm{PAF}=\frac{\mathrm{p} \cdot(\mathrm{RR}-1)}{\mathrm{p} \cdot(\mathrm{RR}-1)+1}
$$

The $95 \%$ confidence intervals (CIs) of PAF were calculated by a Delta method ${ }^{22}$, assuming that $\ln (\mathrm{RR})$ has a normal distribution:

$$
\operatorname{var}(\mathrm{PAF})=\frac{(\mathrm{RR}-1)^{2} \cdot \operatorname{var}(\mathrm{p})+(\mathrm{p} \cdot \mathrm{RR})^{2} \cdot \operatorname{var}[\ln (\mathrm{RR})]}{[\mathrm{p} \cdot(\mathrm{RR}-1)+1]^{4}}
$$

Finally, the overall PAF in each province was calculated by dividing the number of estimated smoking-attributable cancer deaths by the total number of cancer deaths among persons aged $\geq 30$ years, in each province.

\section{RESULTS}

In 2013, smoking contributed to a total of 421566 cancer deaths $(19.46 \%$ of all cancer deaths) in mainland China, with 400701 of these deaths occurring in men (29.34\%) and 21368 (2.67\%) in women (Table 2 ).

For the sexes combined, the faction of cancer deaths attributable to smoking was highest in Inner Mongolia, Chongqing, Jilin and Guizhou, and PAF ranged from $22.46 \%$ to $23.50 \%$ (Table 2 ; and Supplementary file, Figure S1). However, the patterns of PAFs were different between men and women, ranging from $15.56 \%$ (95\% CI: $9.12-21.82 \%$ ) in Tibet to $35.09 \%$ (95\% CI: $25.68-45.83 \%$ ) in Guizhou among men, and from $0.28 \%$ (95\% CI: $0.00-0.64 \%$ ) in Hainan to $10.44 \%$ (95\% CI: 4.86-16.32\%) in Jilin among women (Table 2). In men, the top 4 PAFs ranged from $35.09 \%$ to $31.65 \%$, for provinces in the Southwest and Northeast regions including Guizhou, Chongqing, Hubei, and Jilin. In women, however, the topmost 4 provinces (Jilin, Tianjin, Inner Mongolia, and Heilongjiang) were located in Northern China, and $\mathrm{PAF}$ ranged from $8.58 \%$ to $10.44 \%$, which was three-fold of the national average (Figure 1).

We also present the site-specific smoking-attributable cancer deaths and the corresponding proportions in 31 provinces among men and women (Figures 2 and 3; and Supplementary file, Figures S2 and S3). Overall, cancers of lung and liver were the two main causes of smokingrelated cancer deaths for both men and women. For men, stomach cancer was the third cause of smokingrelated cancer deaths, followed by cancers of esophagus, mouth/larynx/pharynx, nasopharynx, bladder, colorectum, pancreas, and kidney. For women, colorectal cancer was the third cause of smoking-related cancer deaths, followed by cancers of pancreas, esophagus, stomach, mouth/larynx/pharynx, nasopharynx, bladder, and kidney. The number and proportion of site-specific smoking-attributable cancer deaths varied substantially across provinces. 
Table 2. Population (N), smoking prevalence (\%), cancer deaths (CD), number and proportion of smoking attributable cancer deaths (SACD), China 2013

\begin{tabular}{|c|c|c|c|c|c|c|c|}
\hline Region & Province & $\mathbf{N}$ & $\%$ & CD & $S A C D$ & $\begin{array}{c}\text { PAF } \\
{ }_{0}^{\circ}(95 \% \text { CI })\end{array}$ & $\begin{array}{l}\text { PAF } \\
\text { rank }\end{array}$ \\
\hline \multicolumn{8}{|l|}{ Men } \\
\hline \multirow[t]{5}{*}{ Northern } & Beijing & 10126430 & 66.82 & 20426 & 5967 & $29.21(19.89-41.19)$ & 16 \\
\hline & Tianjin & 6907091 & 59.71 & 13046 & 4066 & $31.17(21.85-42.44)$ & 6 \\
\hline & Hebei & 36430286 & 63.41 & 65439 & 18165 & $27.76(18.25-39.88)$ & 22 \\
\hline & Shanxi & 18338760 & 64.97 & 33416 & 9968 & $29.83(18.79-44.60)$ & 13 \\
\hline & Inner Mongolia & 12838243 & 69.28 & 24398 & 7494 & $30.72(19.79-45.18)$ & 7 \\
\hline \multirow[t]{3}{*}{ Northeast } & Liaoning & 22147745 & 54.70 & 53673 & 15198 & $28.32(18.72-40.34)$ & 21 \\
\hline & Jilin & 13907218 & 64.39 & 24847 & 7863 & $31.65(22.20-42.76)$ & 4 \\
\hline & Heilongjiang & 19426106 & 59.76 & 43269 & 13173 & $30.44(21.05-42.01)$ & 9 \\
\hline \multirow[t]{7}{*}{ Eastern } & Shanghai & 11854916 & 64.90 & 38578 & 9949 & $25.79(17.07-37.01)$ & 26 \\
\hline & Jiangsu & 39626707 & 61.78 & 79512 & 21920 & 27.57 (16.66-42.67) & 23 \\
\hline & Zhejiang & 27965641 & 63.22 & 65728 & 20006 & $30.44(20.72-42.70)$ & 9 \\
\hline & Anhui & 30245513 & 62.02 & 67127 & 20583 & $30.66(19.11-45.71)$ & 8 \\
\hline & Fujian & 18981054 & 61.47 & 41173 & 10898 & $26.47(16.21-40.01)$ & 24 \\
\hline & Jiangxi & 23003521 & 62.96 & 37340 & 10922 & $29.25(19.99-40.20)$ & 15 \\
\hline & Shandong & 48446944 & 55.78 & 119352 & 34230 & $28.68(18.27-42.41)$ & 18 \\
\hline \multirow[t]{3}{*}{ Central } & Henan & 47493063 & 63.10 & 90311 & 26199 & $29.01(17.95-43.82)$ & 17 \\
\hline & Hubei & 29391247 & 65.03 & 64673 & 20533 & $31.75(21.88-43.75)$ & 3 \\
\hline & Hunan & 33776459 & 63.72 & 52206 & 16417 & $31.45(22.40-41.67)$ & 5 \\
\hline \multirow[t]{3}{*}{ Southern } & Guangdong & 54400538 & 66.04 & 92992 & 28205 & $30.33(20.67-41.82)$ & 12 \\
\hline & Guangxi & 23924704 & 57.54 & 47584 & 13515 & $28.40(19.20-39.04)$ & 19 \\
\hline & Hainan & 4592283 & 54.30 & 8876 & 2112 & $23.79(15.63-32.88)$ & 30 \\
\hline \multirow[t]{5}{*}{ Southwest } & Chongqing & 14608870 & 66.66 & 34925 & 12164 & $34.83(23.38-49.80)$ & 2 \\
\hline & Sichuan & 40827834 & 63.38 & 95297 & 28125 & $29.51(18.48-44.24)$ & 14 \\
\hline & Guizhou & 17905471 & 73.82 & 23344 & 8191 & $35.09(25.68-45.83)$ & 1 \\
\hline & Yunnan & 23856696 & 75.48 & 35566 & 10803 & $30.37(21.41-41.41)$ & 11 \\
\hline & Tibet & 1542652 & 47.67 & 559 & 87 & $15.56(9.12-21.82)$ & 31 \\
\hline \multirow[t]{6}{*}{ Northwest } & Shaanxi & 19287575 & 63.50 & 35344 & 10014 & $28.33(16.88-44.28)$ & 20 \\
\hline & Gansu & 13064193 & 62.76 & 32955 & 7968 & $24.18(13.48-38.87)$ & 29 \\
\hline & Qinghai & 2913793 & 67.45 & 4078 & 1048 & $25.70(16.14-37.96)$ & 27 \\
\hline & Ningxia & 3227404 & 59.38 & 4602 & 1213 & $26.36(17.08-38.16)$ & 25 \\
\hline & Xinjiang & 11270147 & 50.36 & 14917 & 3705 & $24.84(16.06-36.03)$ & 28 \\
\hline & National & 682329104 & & 1365553 & 400701 & $29.34(19.21-42.31)$ & \\
\hline \multicolumn{8}{|l|}{ Women } \\
\hline \multirow[t]{5}{*}{ Northern } & Beijing & 9485938 & 6.93 & 14279 & 670 & 4.69 (1.90-7.71) & 5 \\
\hline & Tianjin & 6031602 & 11.56 & 9599 & 901 & $9.39(4.49-14.52)$ & 2 \\
\hline & Hebei & 35423924 & 5.55 & 39607 & 1431 & $3.61(1.51-5.85)$ & 6 \\
\hline & Shanxi & 17373341 & 2.56 & 18375 & 285 & $1.55(0.55-2.67)$ & 21 \\
\hline & Inner Mongolia & 11868048 & 12.50 & 12667 & 1182 & $9.33(4.19-14.77)$ & 3 \\
\hline \multirow[t]{3}{*}{ Northeast } & Liaoning & 21598578 & 5.45 & 47353 & 1550 & $3.27(1.29-5.35)$ & 8 \\
\hline & Jilin & 13545597 & 14.91 & 16577 & 1730 & $10.44(4.86-16.32)$ & 1 \\
\hline & Heilongjiang & 18887885 & 10.67 & 29662 & 2544 & $8.58(3.78-13.64)$ & 4 \\
\hline
\end{tabular}


Table 2. Continued

\begin{tabular}{|c|c|c|c|c|c|c|c|}
\hline Region & Province & $\mathbf{N}$ & $\%$ & $\mathrm{CD}$ & $S A C D$ & $\begin{array}{c}\text { PAF } \\
{ }_{0}(95 \% \text { CI })\end{array}$ & $\begin{array}{l}\text { PAF } \\
\text { rank }\end{array}$ \\
\hline \multirow[t]{7}{*}{ Eastern } & Shanghai & 11164280 & 2.09 & 27631 & 324 & $1.17(0.29-2.15)$ & 25 \\
\hline & Jiangsu & 39034234 & 3.53 & 45097 & 1002 & $2.22(0.81-3.75)$ & 13 \\
\hline & Zhejiang & 26461250 & 1.90 & 35320 & 481 & $1.36(0.42-2.37)$ & 24 \\
\hline & Anhui & 29254955 & 4.08 & 33289 & 811 & $2.44(0.89-4.13)$ & 10 \\
\hline & Fujian & 17913163 & 0.82 & 18249 & 89 & $0.49(0.10-0.91)$ & 30 \\
\hline & Jiangxi & 21564276 & 2.42 & 19975 & 308 & $1.54(0.48-2.67)$ & 22 \\
\hline & Shandong & 47345775 & 2.73 & 66521 & 1448 & $2.18(0.85-3.58)$ & 14 \\
\hline \multirow[t]{3}{*}{ Central } & Henan & 46536876 & 1.39 & 61735 & 549 & $0.89(0.29-1.53)$ & 28 \\
\hline & Hubei & 27846480 & 2.71 & 33938 & 651 & $1.92(0.67-3.29)$ & 18 \\
\hline & Hunan & 31924303 & 3.11 & 28374 & 575 & $2.03(0.72-3.43)$ & 16 \\
\hline \multirow[t]{3}{*}{ Southern } & Guangdong & 49919921 & 2.54 & 53371 & 1023 & $1.92(0.62-3.28)$ & 18 \\
\hline & Guangxi & 22099057 & 2.03 & 22450 & 331 & $1.47(0.46-2.57)$ & 23 \\
\hline & Hainan & 4079202 & 0.53 & 4657 & 13 & $0.28(0.00-0.64)$ & 31 \\
\hline \multirow[t]{5}{*}{ Southwest } & Chongqing & 14237300 & 2.88 & 18652 & 428 & $2.29(0.88-3.80)$ & 12 \\
\hline & Sichuan & 39589694 & 2.77 & 49805 & 974 & $1.96(0.69-3.30)$ & 17 \\
\hline & Guizhou & 16843085 & 2.76 & 14640 & 340 & $2.32(0.75-4.00)$ & 11 \\
\hline & Yunnan & 22110070 & 3.65 & 24683 & 504 & $2.04(0.66-3.52)$ & 15 \\
\hline & Tibet & 1459513 & 6.97 & 399 & 4 & $1.00(0.25-2.01)$ & 27 \\
\hline \multirow[t]{6}{*}{ Northwest } & Shaanxi & 18039804 & 1.94 & 19929 & 222 & $1.11(0.39-1.90)$ & 26 \\
\hline & Gansu & 12511070 & 1.67 & 19858 & 151 & $0.76(0.24-1.36)$ & 29 \\
\hline & Qinghai & 2712930 & 2.90 & 2366 & 37 & $1.56(0.42-2.92)$ & 20 \\
\hline & Ningxia & 3073946 & 4.93 & 2536 & 84 & $3.31(1.26-5.60)$ & 7 \\
\hline & Xinjiang & 10545668 & 3.85 & 8993 & 223 & $2.48(0.88-4.24)$ & 9 \\
\hline & National & 650481765 & & 800587 & 20865 & $2.61(1.01-4.31)$ & \\
\hline \multicolumn{8}{|l|}{ Total } \\
\hline \multirow[t]{5}{*}{ Northern } & Beijing & 19612368 & 37.85 & 34705 & 6637 & $19.12(12.49-27.41)$ & 17 \\
\hline & Tianjin & 12938693 & 37.26 & 22645 & 4967 & $21.93(14.49-30.61)$ & 5 \\
\hline & Hebei & 71854210 & 34.88 & 105046 & 19596 & $18.65(11.94-27.05)$ & 19 \\
\hline & Shanxi & 35712101 & 34.61 & 51791 & 10253 & $19.80(12.32-29.72)$ & 13 \\
\hline & Inner Mongolia & 24706291 & 42.00 & 37065 & 8676 & $23.41(14.46-34.79)$ & 2 \\
\hline \multirow[t]{3}{*}{ Northeast } & Liaoning & 43746323 & 30.39 & 101026 & 16748 & $16.58(10.55-23.94)$ & 26 \\
\hline & Jilin & 27452815 & 39.97 & 41424 & 9593 & $23.16(15.26-32.18)$ & 3 \\
\hline & Heilongjiang & 38313991 & 35.56 & 72931 & 15717 & $21.55(14.03-30.47)$ & 6 \\
\hline \multirow[t]{7}{*}{ Eastern } & Shanghai & 23019196 & 34.43 & 66209 & 10273 & $15.52(10.07-22.46)$ & 29 \\
\hline & Jiangsu & 78660941 & 32.88 & 124609 & 22922 & 18.40 (10.92-28.58) & 22 \\
\hline & Zhejiang & 54426891 & 33.41 & 101048 & 20487 & $20.27(13.62-28.60)$ & 10 \\
\hline & Anhui & 59500468 & 33.54 & 100416 & 21394 & $21.31(13.07-31.93)$ & 8 \\
\hline & Fujian & 36894217 & 32.02 & 59422 & 10987 & $18.49(11.26-28.00)$ & 21 \\
\hline & Jiangxi & 44567797 & 33.66 & 57315 & 11230 & $19.59(13.19-27.12)$ & 15 \\
\hline & Shandong & 95792719 & 29.56 & 185873 & 35678 & $19.19(12.03-28.52)$ & 16 \\
\hline \multirow[t]{3}{*}{ Central } & Henan & 94029939 & 32.56 & 152046 & 26748 & 17.59 (10.78-26.65) & 24 \\
\hline & Hubei & 57237727 & 34.71 & 98611 & 21184 & $21.48(14.58-29.83)$ & 7 \\
\hline & Hunan & 65700762 & 34.27 & 80580 & 16992 & 21.09 (14.77-28.21) & 9 \\
\hline
\end{tabular}


Table 2. Continued

\begin{tabular}{|c|c|c|c|c|c|c|c|}
\hline Region & Province & $\mathbf{N}$ & $\%$ & CD & SACD & $\begin{array}{c}\text { PAF } \\
{ }_{0}\left(95{ }^{\circ} \mathrm{CI}\right)\end{array}$ & $\begin{array}{l}\text { PIF } \\
\text { rank }\end{array}$ \\
\hline \multirow[t]{3}{*}{ Southern } & Guangdong & 104320459 & 35.65 & 146363 & 29228 & $19.97(13.36-27.77)$ & 12 \\
\hline & Guangxi & 46023761 & 30.89 & 70034 & 13846 & $19.77(13.20-27.35)$ & 14 \\
\hline & Hainan & 8671485 & 28.07 & 13533 & 2125 & $15.70(10.25-21.78)$ & 28 \\
\hline \multirow[t]{5}{*}{ Southwest } & Chongqing & 28846170 & 35.83 & 53577 & 12592 & $23.50(15.55-33.79)$ & 1 \\
\hline & Sichuan & 80417528 & 34.87 & 145102 & 29099 & $20.05(12.37-30.19)$ & 11 \\
\hline & Guizhou & 34748556 & 38.75 & 37984 & 8531 & $22.46(16.07-29.71)$ & 4 \\
\hline & Yunnan & 45966766 & 40.12 & 60249 & 11307 & $18.77(12.91-25.89)$ & 18 \\
\hline & Tibet & 3002165 & 27.94 & 958 & 91 & $9.50(5.43-13.57)$ & 31 \\
\hline \multirow[t]{6}{*}{ Northwest } & Shaanxi & 37327379 & 33.89 & 55273 & 10236 & $18.52(10.93-29.00)$ & 20 \\
\hline & Gansu & 25575263 & 33.06 & 52813 & 8119 & 15.37 (8.50-24.77) & 30 \\
\hline & Qinghai & 5626723 & 36.25 & 6444 & 1085 & $16.84(10.37-25.09)$ & 25 \\
\hline & Ningxia & 6301350 & 32.74 & 7138 & 1297 & $18.17(11.46-26.59)$ & 23 \\
\hline & Xinjiang & 21815815 & 27.93 & 23910 & 3928 & $16.43(10.35-24.07)$ & 27 \\
\hline & National & 1332810869 & & 2166140 & 421566 & $19.46(12.49-28.26)$ & \\
\hline
\end{tabular}

$\mathrm{Cl}$ : confidence interval. PAF: population attributable fraction. SACD: smoking attributable cancer deaths.

Figure 1. Rank of smoking attributable cancer mortality in 2013 by gender
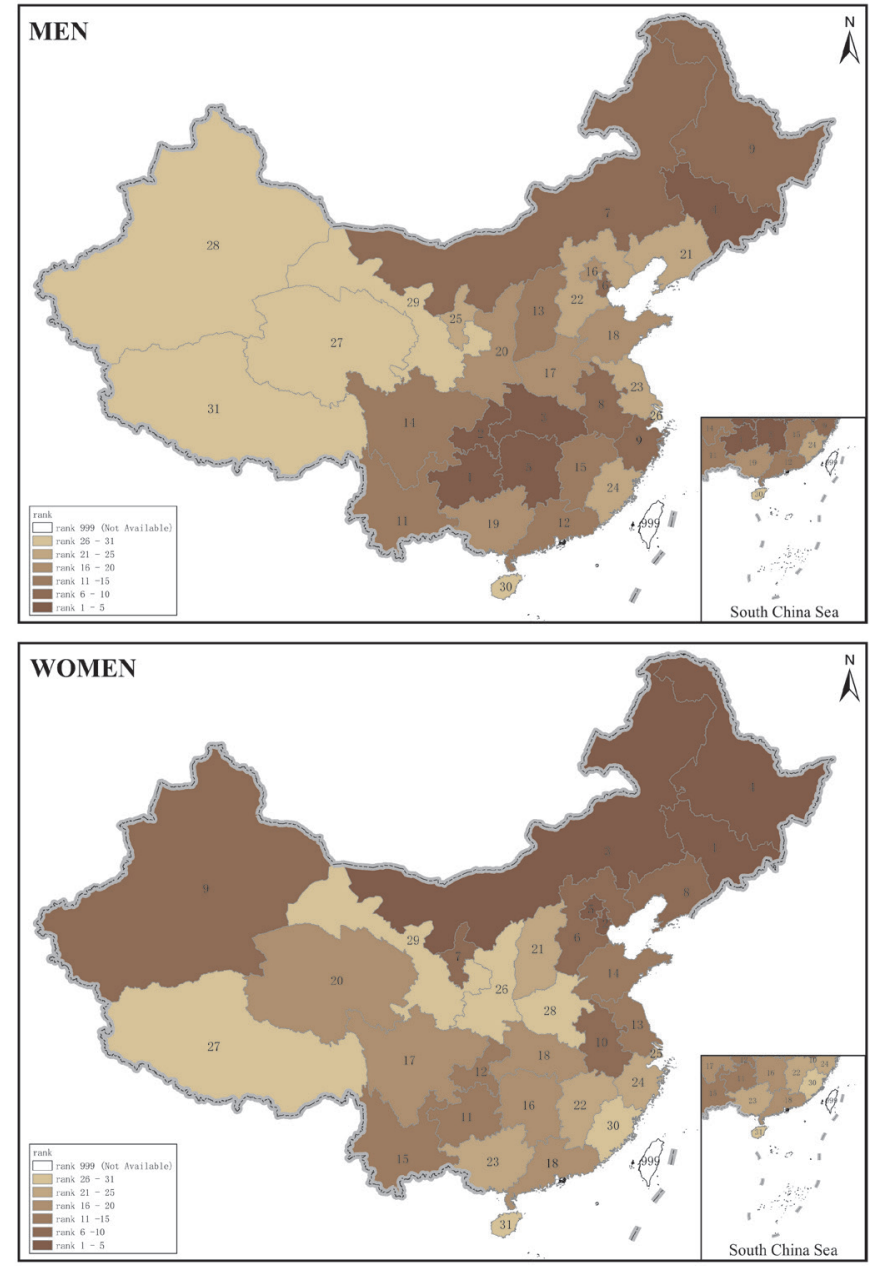

Tob. Induc. Dis. 2020;18(June):49 
Figure 2. Heatmap of site- specific cancer deaths in the 31 provinces of mainland China in 2013 in men MEN

\begin{tabular}{|c|c|c|c|c|c|c|c|c|c|c|c|}
\hline Provinces & $\begin{array}{l}\text { Oral/Larynx } \\
\text { / Pharynx }\end{array}$ & Esophagus & Stomach & Colorectum & Liver & Pancreas & Lung & Bladder & $\begin{array}{c}\text { Naso- } \\
\text { pharynx }\end{array}$ & Kidney & Total \\
\hline Beijing & 175 & 314 & 397 & 154 & 448 & 102 & 4078 & 230 & 35 & 34 & 5967 \\
\hline Tianjin & 117 & 125 & 235 & 72 & 300 & 52 & 3000 & 113 & 32 & 20 & 4066 \\
\hline Hebei & 270 & 1665 & 2789 & 215 & 1671 & 141 & 10988 & 299 & 92 & 35 & 18165 \\
\hline Shanxi & 222 & 1257 & 1726 & 147 & 783 & 92 & 5556 & 128 & 42 & 15 & 9968 \\
\hline Inner Mongolia & 220 & 824 & 648 & 134 & 1050 & 85 & 4374 & 90 & 44 & 25 & 7494 \\
\hline Liaoning & 373 & 691 & 1104 & 586 & 1435 & 191 & 10290 & 393 & 96 & 39 & 15198 \\
\hline Jilin & 183 & 197 & 652 & 151 & 963 & 88 & 5289 & 250 & 68 & 22 & 7863 \\
\hline Heilongjiang & 386 & 486 & 869 & 256 & 1395 & 185 & 9207 & 285 & 48 & 56 & 13173 \\
\hline Shanghai & 333 & 368 & 1077 & 388 & 680 & 224 & 6145 & 434 & 235 & 65 & 9949 \\
\hline Jiangsu & 344 & 3208 & 3037 & 309 & 2263 & 306 & 11795 & 362 & 263 & 33 & 21920 \\
\hline Zhejiang & 322 & 1085 & 1839 & 367 & 1951 & 316 & 13333 & 374 & 370 & 49 & 20006 \\
\hline Anhui & 299 & 2323 & 3227 & 273 & 3402 & 160 & 10401 & 255 & 208 & 35 & 20583 \\
\hline Fujian & 263 & 1344 & 1357 & 206 & 1607 & 75 & 5575 & 131 & 321 & 19 & 10898 \\
\hline Jiangxi & 259 & 440 & 1076 & 192 & 1519 & 69 & 6689 & 180 & 460 & 38 & 10922 \\
\hline Shandong & 567 & 3757 & 4174 & 408 & 3066 & 240 & 21126 & 624 & 204 & 64 & 34230 \\
\hline Henan & 404 & 3635 & 3634 & 329 & 2898 & 148 & 14464 & 431 & 207 & 49 & 26199 \\
\hline Hubei & 595 & 1033 & 1505 & 342 & 2252 & 190 & 13632 & 545 & 383 & 56 & 20533 \\
\hline Hunan & 592 & 412 & 956 & 229 & 1760 & 74 & 11320 & 277 & 768 & 29 & 16417 \\
\hline Guangdong & 1184 & 1110 & 1047 & 718 & 3614 & 211 & 17453 & 626 & 2167 & 75 & 28205 \\
\hline Guangxi & 373 & 479 & 747 & 234 & 2191 & 61 & 8324 & 251 & 825 & 30 & 13515 \\
\hline Hainan & 119 & 32 & 139 & 59 & 405 & 6 & 1141 & 97 & 105 & 9 & 2112 \\
\hline Chongqing & 279 & 1211 & 527 & 195 & 995 & 98 & 8462 & 188 & 190 & 19 & 12164 \\
\hline Sichuan & 669 & 3510 & 2477 & 531 & 3052 & 239 & 16447 & 618 & 546 & 36 & 28125 \\
\hline Guizhou & 270 & 100 & 388 & 172 & 909 & 77 & 5757 & 168 & 334 & 16 & 8191 \\
\hline Yunnan & 385 & 292 & 615 & 355 & 1162 & 190 & 7256 & 372 & 144 & 32 & 10803 \\
\hline Tibet & 0 & 0 & 41 & 0 & 46 & 0 & 0 & 0 & 0 & 0 & 87 \\
\hline Shaanxi & 139 & 1744 & 1682 & 90 & 825 & 73 & 5253 & 122 & 65 & 21 & 10014 \\
\hline Gansu & 89 & 1415 & 2509 & 140 & 677 & 55 & 2833 & 171 & 69 & 10 & 7968 \\
\hline Qinghai & 16 & 96 & 226 & 21 & 163 & 12 & 480 & 25 & 5 & 4 & 1048 \\
\hline Ningxia & 35 & 91 & 219 & 15 & 127 & 15 & 666 & 26 & 16 & 3 & 1213 \\
\hline Xinjiang & 89 & 240 & 344 & 68 & 379 & 50 & 2395 & 98 & 27 & 15 & 3705 \\
\hline Total & 9571 & 33484 & 41263 & 7356 & 43988 & 3825 & 243729 & 8163 & 8369 & 953 & 400701 \\
\hline
\end{tabular}

Figure 3. Heatmap of site-specific cancer deaths in the 31 provinces of mainland China in 2013 in women WOMEN

\begin{tabular}{|c|c|c|c|c|c|c|c|c|c|c|c|}
\hline Provinces & $\begin{array}{l}\text { Oral/Larynx } \\
\text { / Pharynx }\end{array}$ & Esophagus & Stomach & Colorectum & Liver & Pancreas & Lung & Bladder & $\begin{array}{c}\text { Naso- } \\
\text { pharynx }\end{array}$ & Kidney & Total \\
\hline Beijing & 10 & 7 & 8 & 42 & 42 & 33 & 516 & 6 & 3 & 3 & 670 \\
\hline Tianjin & 13 & 6 & 9 & 33 & 51 & 30 & 748 & 5 & 4 & 2 & 901 \\
\hline Hebei & 14 & 69 & 47 & 47 & 147 & 31 & 1067 & 5 & 3 & 1 & 1431 \\
\hline Shanxi & 3 & 22 & 10 & 9 & 38 & 7 & 192 & 2 & 2 & 0 & 285 \\
\hline Inner Mongolia & 9 & 13 & 16 & 45 & 135 & 49 & 899 & 6 & 7 & 3 & 1182 \\
\hline Liaoning & 18 & 8 & 20 & 79 & 124 & 53 & 1231 & 7 & 7 & 3 & 1550 \\
\hline Jilin & 17 & 8 & 23 & 73 & 203 & 51 & 1331 & 13 & 6 & 5 & 1730 \\
\hline Heilongjiang & 26 & 13 & 28 & 121 & 242 & 112 & 1972 & 18 & 6 & 6 & 2544 \\
\hline Shanghai & 5 & 3 & 8 & 36 & 25 & 25 & 213 & 3 & 5 & 1 & 324 \\
\hline Jiangsu & 11 & 78 & 32 & 44 & 125 & 55 & 642 & 4 & 10 & 1 & 1002 \\
\hline Zhejiang & 7 & 7 & 11 & 26 & 54 & 31 & 336 & 2 & 6 & 1 & 481 \\
\hline Anhui & 11 & 46 & 34 & 41 & 97 & 36 & 534 & 3 & 8 & 1 & 811 \\
\hline Fujian & 1 & 6 & 3 & 6 & 14 & 2 & 55 & 0 & 2 & 0 & 89 \\
\hline Jiangxi & 7 & 4 & 9 & 18 & 42 & 5 & 214 & 1 & 7 & 1 & 308 \\
\hline Shandong & 12 & 55 & 33 & 52 & 141 & 34 & 1109 & 5 & 5 & 2 & 1448 \\
\hline Henan & 9 & 45 & 17 & 20 & 75 & 11 & 366 & 2 & 3 & 1 & 549 \\
\hline Hubei & 9 & 11 & 11 & 32 & 81 & 23 & 471 & 4 & 8 & 1 & 651 \\
\hline Hunan & 7 & 5 & 10 & 27 & 76 & 9 & 411 & 3 & 26 & 1 & 575 \\
\hline Guangdong & 17 & 5 & 12 & 71 & 84 & 29 & 749 & 5 & 50 & 1 & 1023 \\
\hline Guangxi & 4 & 6 & 5 & 19 & 43 & 5 & 232 & 1 & 16 & 0 & 331 \\
\hline Hainan & 0 & 0 & 0 & 1 & 2 & 0 & 9 & 0 & 1 & 0 & 13 \\
\hline Chongqing & 4 & 19 & 5 & 20 & 43 & 12 & 320 & 1 & 3 & 1 & 428 \\
\hline Sichuan & 11 & 39 & 24 & 51 & 130 & 28 & 671 & 3 & 16 & 1 & 974 \\
\hline Guizhou & 4 & 0 & 4 & 14 & 53 & 11 & 241 & 2 & 11 & 0 & 340 \\
\hline Yunnan & 8 & 3 & 8 & 47 & 63 & 23 & 338 & 3 & 10 & 1 & 504 \\
\hline Tibet & 0 & 2 & 2 & 0 & 0 & 0 & 0 & 0 & 0 & 0 & 4 \\
\hline Shaanxi & 3 & 18 & 7 & 6 & 31 & 7 & 147 & 1 & 1 & 1 & 222 \\
\hline Gansu & 2 & 12 & 9 & 7 & 20 & 3 & 94 & 1 & 3 & 0 & 151 \\
\hline Qinghai & 0 & 1 & 1 & 2 & 9 & 2 & 22 & 0 & 0 & 0 & 37 \\
\hline Ningxia & 1 & 3 & 2 & 2 & 9 & 2 & 64 & 0 & 1 & 0 & 84 \\
\hline Ximjiang & 1 & 6 & 4 & 9 & 32 & 11 & 155 & 2 & 2 & 1 & 223 \\
\hline Total & 244 & 520 & 412 & 1000 & 2231 & 730 & 15349 & 108 & 232 & 39 & 20865 \\
\hline
\end{tabular}




\section{DISCUSSION}

This study provides a systematic evidence-based assessment of province-specific smoking-attributable cancer burden in China. Smoking-attributable cancer mortality in men (PAF=29.34\%; 95\%CI: $19.21-$ $42.31 \%$ ) was substantially higher than that in women (PAF=2.61\%; 95\%CI: 1.01-4.31\%), which is mainly explained by the higher smoking prevalence in men. Overall, smoking was responsible for approximately $20 \%$ in all cancer deaths in mainland China, but this proportion of smoking-attributable cancer deaths varied substantially across provinces.

Regional variation in PAFs can be primarily explained by differences in smoking prevalence, which has prevailed partly due to variations in tobacco control policies and programs, socioeconomic status and education, culture, and attitudes. Since China ratified the WHO Framework Convention on Tobacco Control in 2005, some metropolitan cities have adopted smoke-free laws prohibiting smoking in public areas. However, the implementation and compliance of smoke-free policy has shown large differences on the subnational leve ${ }^{23}$. Tobacco control policies are heavily influenced by the tobacco industry in all provinces, and tobacco use prevalences are higher in the cities near where tobacco is grown, such as in the Yunnan and Guizhou provinces. However, higher smoking prevalences in the Northeast regions, with nearly no tobacco plantations, can be explained by low smoking cessation rates and low cost of manufactured cigarettes there ${ }^{24}$. Moreover, low socioeconomic status is considered to be associated with higher smoking prevalence and lower smoking cessation rates ${ }^{25,26}$. People with lower educational levels are less aware of the adverse effects of smoking. Generally, cigarettes are a popular 'social currency' in China, especially in the rural regions ${ }^{27,28}$.

The larger burden of smoking-related cancer deaths in men than in women most likely reflects the higher prevalence of smoking among men than in women, but there are large discrepancies in PAFs among women across provinces. PAFs among women living in the Southwest provinces were approximately $2-3 \%$, which were lower than the national average, compared with that in men of Southwest China. Moreover, tobacco control policies and programmes appeared not to be well implemented in Northern China. The topmost 4 provinces (Jilin, Tianjin, Inner
Mongolia, and Heilongjiang) had PAFs that were three-fold higher than the national average among women, and the top nine provinces with higher PAFs were all located north of the Yangtze River.

The order of site-specific cancer deaths in China has changed in the past decades ${ }^{29}$, but lung cancer have ranked as first in the smoking-attributable cancer deaths for both genders, consistent with the results from other developing countries ${ }^{30}$. The tobacco epidemic was responsible for the rapid increase in lung cancer mortality in recent decades ${ }^{31}$. However, in some provinces, we found that smoking contributed more deaths from cancers of the stomach and esophagus than liver cancer among men, which was driven by the higher mortality rates of stomach and esophageal cancers in these regions.

Our province-specific PAF estimates were comparable with those from the limited evidence available from previous studies in China based on similar methodology. In our study, estimates of provincial PAFs for smoking and cancer deaths in men were higher than the corresponding figures of a previous study ${ }^{32}$. This discrepancy can be primarily explained by different sources of smoking prevalence. Our study used the smoking prevalence in 1998 as the exposure rate, which was higher than the figures of 2002 used in the Xia et al. ${ }^{32}$ study, based on a different assumption of the latency time. Although the smoking definitions were similar in both studies, there were some differences in how PAF values were estimated. For smoking-related cancers, we included 10 cancer sites in our study versus 5 major cancer sites together with other minor sites in the Xia et al. ${ }^{32}$ study. In addition, the estimates of smoking attributable cancer mortality could be affected by the time fluctuation of cancer registered data and different sources of RRs. For most cancers, RRs used in our estimates were abstracted from the results of the Chinese population in a pooled analysis of 21 cohort studies in Asia, which were relatively lower than those in the Western population ${ }^{33,34}$, but higher than those in the Xia et al. ${ }^{32}$ study. In their study, RRs were taken from the China Kadoorie Biobank Study from 10 regions during 7 years of follow-up ${ }^{35}$, which might be underestimated due to the short period of follow-up. Regardless of the PAF disparities, the order of provincial PAFs was still comparable in the two studies (Supplementary file, Figure S4). 
In China, smoking prevalence in men declined rapidly during the 1980s and 1990s. Nevertheless, the fraction of smoking-attributable cancer mortality increased slightly over time. The Liu et al. ${ }^{36}$ study in 1990 reported a fraction of $24.4 \%$ in men, but the corresponding figures were $28.0 \%$ and $32.7 \%$ in 2005 in the $\mathrm{Gu}$ et al. ${ }^{12}$ study and the Wang et al. ${ }^{8}$ study, respectively. There might be potential overestimation in relative risk for lung cancer sourced from limited Shanghai residents used in the Wang et al. ${ }^{8}$ study. Methodological differences might be a source of disparity, but potential transition in stages of smokingrelated cancer might be a greater contributor. Moreover, our PAF estimates were comparable with the results from the Western populations ${ }^{37}$, while smoking prevalence was relatively higher in Chinese men $^{7,15,16}$. In China, widespread tobacco smoking began several decades later than in Europe and North America, thus China was at an earlier stage of the tobacco epidemic compared with the developed countries $^{38}$. In recent years, smoking prevalence has declined slowly in China due to enforcement of tobacco control policies, but still remains high, especially in men. The current smoke-free policy is still inadequate in reducing prevalence and affecting smoker's behavior ${ }^{39}$. As the tobacco epidemic grows, smoking associated cancer deaths will be elevated due to the long latency time of smoking related cancers. More effective efforts in tobacco control, including increasing tobacco taxes and maintaining funding of anti-smoking campaigns, are needed to reduce the smoking-related cancer burden.

\section{Strengths and limitations}

A strength of our study is the estimate of site-specific smoking attributable cancer mortality at the provincial level. However, our study has also several limitations and uncertainty. First, in our study, indirect smoking prevalence data were used due to lack of qualified age-specific data on provincial smoking prevalence and smoking prevalence based on self-reported results can be underestimated ${ }^{40}$, which could affect our estimates. Second, only 10 major types of smokingrelated cancers were included in our study, and some other smoking-associated cancers were excluded due to the lack of reliable data, so that our PAF values might be underestimated. Third, secondhand smoking also plays a crucial role in the association of smoking and lung cancer, but it was not included in our study because it is difficult to quantify and there are no corresponding reliable provincial data based on latency time. However, in our previous study, we estimated that $11.1 \%$ of lung cancer deaths among non-smoking women were attributable to involuntary smoking from the spouse or at the workplace ${ }^{10}$, which is very comparable with that of the Xia et al. ${ }^{32}$ study (11.5\%). Finally, in our study, we only considered smoking status as never or ever, and were not able to collect information on the type, starting age, amount and duration of smoking. However, using alternative definitions of smoking may not substantially alter the PAF estimates based on the Xia et al. ${ }^{32}$ study.

\section{CONCLUSIONS}

Our study provides a systematic assessment of province-specific cancer burden of tobacco smoking in China in 2013. We found that smoking was responsible for nearly $20 \%$ of cancer deaths in the Chinese population in 2013, and that the proportion of smoking-attributable cancer deaths varies across provinces. Our findings provide strong evidence for more effective programs and innovative new strategies for local tobacco control to reduce the high burden of smoking-related cancers in the provinces of mainland China.

\section{REFERENCES}

1. Chen W, Zheng R, Baade PD, et al. Cancer statistics in China, 2015. CA Cancer J Clin. 2016;66(2):115-132. doi:10.3322/caac. 21338

2. Chen W, Xia C, Zheng R, et al. Disparities by province, age, and sex in site-specific cancer burden attributable to 23 potentially modifiable risk factors in China: a comparative risk assessment. Lancet Glob Heal. 2019;7(2):e257-e269. doi:10.1016/s2214-109x(18)30488-1

3. Doll R. COMMENTARY: Nature and nurture: possibilities for cancer control. Carcinogenesis. 1996;17(2):177-184. doi:10.1093/carcin/17.2.177

4. World Health Organization, International Agency for Research on Cancer. IARC Monographs on the Evaluation of Carcinogenic Risks to Humans: Volume 83. Tobacco Smoke and Involuntary Smoking. Lyon, France: International Agency for Research on Cancer; 2004.

5. Olsen JH, Andersen A, Dreyer L, et al. Summary of avoidable cancers in the Nordic countries. APMIS. 1997;105(S76):141146. doi:10.1111/j.1600-0463.1997.tb05617.x

6. Ministry of Health People's Republic of China. [China's Smoking Hazard Health Report]. People's Medical Publishing House(PMPH); 2012. http://wsjkw.gxzf.gov. 
cn/uploads/soft/120604/\%E4\%B8\%AD\%E5\%9B\%BD\% E5\%90\%B8\%E7\%83\%9F\%E5\%8D\%B1\%E5\%AE\%B3\%E5\% 81\%A5\%E5\%BA\%B7\%E6\%8A\%A5\%E5\%91\%8A\%E5\%86\% 85\%E5\%AE\%B9\%E6\%A6\%82\%E8\%A6\%81.pdf. Accessed November 22, 2019.

7. Li Q, Hsia J, Yang G. Prevalence of smoking in China in 2010. N Engl J Med. 2011;364(25):2469-2470. doi:10.1056/nejmc1102459

8. Wang JB, Jiang Y, Liang H, et al. Attributable causes of cancer in China. Ann Oncol. 2012;23(11):2983-2989. doi:10.1093/annonc/mds139

9. Lortet-Tieulent J, Sauer AG, Siegel RL, et al. State-Level Cancer Mortality Attributable to Cigarette Smoking in the United States. JAMA Intern Med. 2016;176(12):17921798. doi:10.1001/jamainternmed.2016.6530

10. Wang JB, Jiang Y, Wei WQ, Yang GH, Qiao YL, Boffetta P. Estimation of cancer incidence and mortality attributable to smoking in China. Cancer Causes Control. 2010;21(6):959-965. doi:10.1007/s10552-010-9523-8

11. Liu YN, Liu JM, Liu SW, et al. [Death and impact of life expectancy attributable to smoking in China, 2013]. Zhonghua Liu Xing Bing Xue Za Zhi. 2017;38(8):10051010. doi:10.3760/cma.j.issn.0254-6450.2017.08.002

12. Gu D, Kelly TN, Wu X, et al. Mortality attributable to smoking in China. N Engl J Med. 2009;360(2):150-159. doi:10.1056/nejmsa0802902

13. Islami F, Chen W, Yu XQ, et al. Cancer deaths and cases attributable to lifestyle factors and infections in China, 2013. Ann Oncol. 2017;28(10):2567-2574. doi:10.1093/annonc/mdx342

14. World Health Organization, International Agency for Research on Cancer. IARC Working Group Reports: Volume 3. Attributable causes of cancer in France in the year 2000. International Agency for Research on Cancer; 2007.

15. Yang G, Fan L, Tan J, et al. Smoking in China: Findings of the 1996 National Prevalence Survey. JAMA. 1999;282(13):1247-1253. doi:10.1001/jama.282.13.1247

16. Ma G, Kong L, Luan D. [The Descriptive Analysis of the Smoking Pattern of People in China]. Zhongguo Man Xing Bing Yu Fang Yu Kong Zhi. 2005;13(5):195-199.

17. World Health Organization. Guidelines for the conduct of tobacco-smoking surveys among health professionals: report of a WHO meeting held in Winnipeg, Canada, 7-9 July 1983 in collaboration with UICC and ACS. World Health Organization; 1984.

18. Zheng W, Mclerran DF, Rolland BA, et al. Burden of Total and Cause-Specific Mortality Related to Tobacco Smoking among Adults Aged $\geq 45$ Years in Asia: A Pooled Analysis of 21 Cohorts. Plos Med. 2014;11(4):e1001631. doi:10.1371/journal.pmed.1001631

19. Cumberbatch MG, Rota M, Catto JW, La Vecchia C. The Role of Tobacco Smoke in Bladder and Kidney Carcinogenesis: A Comparison of Exposures and Metaanalysis of Incidence and Mortality Risks. Eur Urol.
2016;70(3):458-466. doi:10.1016/j.eururo.2015.06.042

20. Tran GD, Sun XD, Abnet CC, et al. Prospective study of risk factors for esophageal and gastric cancers in the Linxian general population trial cohort in China. Int J Cancer. 2005;113(3):456-463. doi:10.1002/ijc.20616

21. World Health Organization. Metrics: Population Attributable Fraction (PAF). http://www.who.int/ healthinfo/global_burden_disease/metrics_paf/en/. Accessed November 22, 2019.

22. Klein LR, Row, Peterson \& Co. A textbook of econometrics. 1953.

23. Lin H, Chang C, Liu Z, Zheng Y. Subnational smokefree laws in China. Tob Induc Dis. 2019;17(November). doi:10.18332/tid/112665

24. Liang X. Report of China City Adult Tobacco Survey 2013-14. Atlanta, Georgia, USA: CDC Foundation; 2015.

25. Jamal A, Homa DM, O'Connor E, et al. Current cigarette smoking among adults - United States, 2005-2014. MMWR Morb Mortal Wkly Rep. 2015;64(44):1233-1240. doi:10.15585/mmwr.mm6444a2

26. Droomers M, Huang X, Fu W, Yang Y, Li H, Zheng P. Educational disparities in the intention to quit smoking among male smokers in China: a cross-sectional survey on the explanations provided by the theory of planned behaviour. BMJ Open. 2016;6:e011058. doi:10.1136/bmjopen-2016-011058

27. Astell-Burt T, Zhang M, Feng X, et al. Geographical Inequality in Tobacco Control in China: Multilevel Evidence From 98058 Participants. Nicotine Tob Res. 2018;20(6):755-765. doi:10.1093/ntr/ntx100

28. Hu M, Rich ZC, Luo D, Xiao S. Cigarette sharing and gifting in rural China: a focus group study. Nicotine Tob Res. 2012;14(3):361-367. doi:10.1093/ntr/ntr262

29. Zeng HM, Zheng RS, Zhang SW, Zhao P, He J, Chen WQ. [Trend analysis of cancer mortality in China between 1989 and 2008]. Zhonghua Zhong Liu Za Zhi. 2012;34(7):525531. doi:10.3760/cma.j.issn.0253-3766.2012.07.011

30. Torre L, Bray F, Siegel R, Ferlay J, Lortettieulent J, Jemal A. Global cancer statistics, 2012. CA Cancer J Clin. 2015;65(2):87-108. doi:10.3322/caac.21262

31. Shen X, Wang L, Zhu L. Spatial Analysis of Regional Factors and Lung Cancer Mortality in China, 1973-2013. Cancer Epidemiol Biomarkers Prev. 2017;26(4):569-577. doi:10.1158/1055-9965.epi-16-0922

32. Xia C, Zheng R, Zeng H, et al. Provincial-level cancer burden attributable to active and second-hand smoking in China. Tob Control. 2019;28(6):669-675. doi:10.1136/tobaccocontrol-2018-054583

33. Thun MJ, Carter BD, Feskanich D, et al. 50-year trends in smoking-related mortality in the United States. N Engl J Med. 2013;368(4):351-364. doi:10.1056/nejmsa1211127

34. Gandini S, Botteri E, Iodice S, et al. Tobacco smoking and cancer: a meta-analysis. Int J Cancer. 2008;122(1):155164. doi:10.1002/ijc.23033

35. Chen ZM, Peto R, Iona A, et al. Emerging tobacco-related 
cancer risks in China: A nationwide, prospective study of 0.5 million adults. Cancer. 2015;121(Suppl 17): 30973106. doi:10.1002/cncr.29560

36. Liu BQ, Peto R, Chen ZM, et al. Emerging tobacco hazards in China: 1. Retrospective proportional mortality study of one million deaths. BMJ. 1998;317(7170):1411-1422. doi:10.1136/bmj.317.7170.1411

37. Cao B, Hill C, Bonaldi C, et al. Cancers attributable to tobacco smoking in France in 2015. Eur J Public Health. 2018;28(4):707-712. doi:10.1093/eurpub/cky077

38. Thun M, Peto R, Boreham J, Lopez AD. Stages of the cigarette epidemic on entering its second century. Tob Control. 2012;21(2):96-101. doi:10.1136/tobaccocontrol-2011-050294

39. Qian X, Gu H, Wang L, et al. Changes in smoking prevalence after the enforcement of smoking control regulations in urban Shanghai, China: Findings from two cross-sectional surveys. Tob Induc Dis. 2018;16(June). doi:10.18332/tid/91095

40. Connor Gorber S, Schofield-Hurwitz S, Hardt J, Levasseur G, Tremblay M. The accuracy of self-reported smoking: a systematic review of the relationship between selfreported and cotinine-assessed smoking status. Nicotine Tob Res. 2009;11(1):12-24. doi:10.1093/ntr/ntn010
ACKNOWLEDGEMENTS

We would like to express our gratitude to the staff of the cancer registries, national cancer centers, and China CDC.

\section{CONFLICTS OF INTEREST}

The authors have completed and submitted the ICMJE Form for Disclosure of Potential Conflicts of Interest and none was reported.

\section{FUNDING}

This work was funded by Zhejiang Provincial Natural Science Foundation of China under Grant No. LY16H260002. The sponsor of the study had no role in study design, data collection, data analysis, data interpretation, decision to publish or preparation of the manuscript.

\section{AUTHORS' CONTRIBUTIONS}

JW and XC conceived and designed the study. LY led the data analysis, wrote the first draft and drafted the final manuscript. LY and JC collected and organized the data. JC and JW contributed to the development of analysis plans, reviewed the results and contributed to the preparation of the final manuscript. JC, XC and JW checked the analysis process and reviewed results. $\mathrm{LY}$ and JC contributed equally to this work. All authors discussed the results, commented on the manuscript and approved the final version.

PROVENANCE AND PEER REVIEW

Not commissioned; externally peer reviewed. 